\title{
Resistive wall impedance in elliptical multilayer vacuum chambers
}

\author{
M. Migliorati๑* and L. Palumbo \\ University of Rome La Sapienza and INFN - Romal, 00185 Rome, Italy \\ C. Zanniniø and N. Biancacci \\ CERN, 1211 Geneva, Switzerland \\ V. G. Vaccaro \\ University of Napoli Federico II, and INFN - Napoli, 80138 Napoli, Italy
}

(Received 15 July 2019; published 12 December 2019)

\begin{abstract}
The resistive wall impedance of a vacuum chamber with elliptic cross section is of particular interest for circular particle accelerators as well as for undulators in free electron lasers. By using the electric field of a point charge and of a small dipole moving at arbitrary speed in an elliptical vacuum chamber, expressed in terms of Mathieu functions, in this paper we take into account the finite conductivity of the beam pipe walls by means of the surface impedance, and evaluate the longitudinal and transverse driving and detuning impedances for any beam velocity. We also extend the definition of the Yokoya form factors, valid in the thick wall regime, at any beam energy, and show that, in the ultra-relativistic limit, they coincide with the ones that are found in literature. The method is also extended to the multilayer vacuum chamber case. Under conditions generally satisfied with particle accelerator beam pipes, the classical transmission line theory can be used to modelling the impedance seen by a bunch in a vacuum chamber with several layers as an equivalent circuit with the same number of load impedances, giving, as result, a surface impedance that can be used in combination with the fields of the elliptic geometry to obtain the resistive wall impedance in an elliptical multilayer vacuum chamber. The results are also compared with a more time consuming 3D electromagnetic code and with solutions for known cases of circular and flat beam pipe.
\end{abstract}

DOI: 10.1103/PhysRevAccelBeams.22.121001

\section{INTRODUCTION}

The coupling impedance [1-5] due to the finite resistivity of the beam vacuum chamber, generally called resistive wall impedance, can represent an important source of impedance, as, for example, in LCLS undulator [6], or even the main contributor, as in the case of the Future Circular Collider project [7-10].

Among several geometries, the elliptic cross section is of particular interest because it can be found in circular machines [11-13] as well as in undulators for free electron lasers [14].

The problem of calculating the coupling impedance of a resistive beam pipe with arbitrary cross section has been derived in the ultrarelativistic limit in Refs. [15-17]. This impedance can be expressed in terms of form factors, known

\footnotetext{
"mauro.migliorati@uniroma1.it
}

Published by the American Physical Society under the terms of the Creative Commons Attribution 4.0 International license. Further distribution of this work must maintain attribution to the author(s) and the published article's title, journal citation, and DOI. as Yokoya form factors, which depend on the ellipticity of the beam pipe and correspond to the ratio between the resistive wall impedance of elliptic shape and the circular one with the radius equal to the minor semiaxis of the ellipse. Moreover, the impedance of an elliptic beam pipe, in the ultrarelativistic limit, has also been addressed in Ref. [14].

The extension to the nonrelativistic case has been obtained in Ref. [18], where, the choice of the field expansions has led to complicated expressions of the electromagnetic field. Another formulation, written as an integral form, has been also derived in Ref. [19] in the classical thick wall regime with a good conductor.

Lastly, there also exist numerical codes that give the beam coupling impedance due to space charge and resistive wall for arbitrary transverse geometries [20].

In this paper we expand the formalism of previous works [21-23], in which we derived the longitudinal and transverse driving and detuning space charge impedances produced by a point charge and a small dipole traveling on the axis of a perfectly conducting elliptic vacuum chamber, by considering the case of a finite resistivity of the beam pipe walls. The resistive wall term is introduced by using the concept of surface impedance, which can also be 


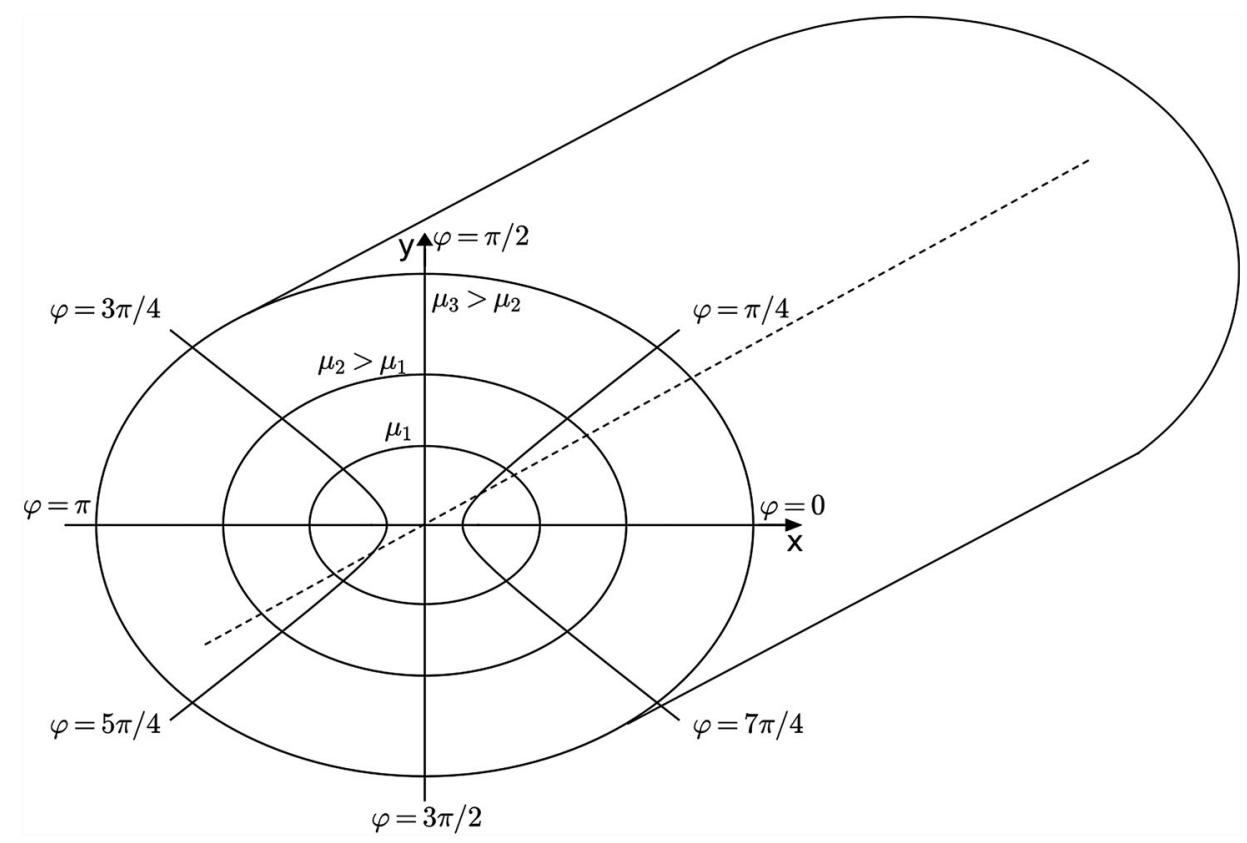

FIG. 1. Elliptical coordinates. The $\varphi$ coordinate describes a series of hyperbolas having the same foci. The $\mu$ coordinate describes confocal ellipses centered in the origin of the coordinate system.

extended to the multilayer case, of interest for many particle accelerators (see, e.g., [8]).

The equations we derive allow to obtain the impedances ranging from the circular shape to the parallel plates, taking into account any value of ellipticity and beam energy.

In the following Sec. II we briefly review the basic equations used to express the electromagnetic fields and the impedances by means of the Mathieu functions, for which we considered, as reference work, the book of McLachlan [24]. Then, in Secs. III and IV, we obtain the longitudinal electric field of a point charge and a dipole including the finite conductivity of the pipe walls. In Sec. V we write the longitudinal and transverse driving and detuning impedances expressed in terms of the surface impedance, and, in Sec. VI, we derive the Yokoya form factors, extending their validity to any beam energy. In the following sections we show the impedances under different conditions. In particular, in Sec. VII we shortly review the surface impedance for simple cases that can be treated analytically, in Sec. VIII we discuss the multilayer vacuum chamber, while some benchmarks with a 3D electromagnetic code are shown in Sec. IX. Finally, Sec. X is dedicated to concluding remarks.

\section{LONGITUDINAL ELECTRIC FIELD IN A PERFECTLY CONDUCTING ELLIPTICAL BEAM PIPE}

In the following we suppose to have a beam pipe with elliptical cross section. For the expansions of the fields and the impedances, we use the transverse elliptical coordinates $\varphi$ and $\mu$, describing respectively a set of hyperbolas having the same foci, and a set of confocal ellipses, as shown in Fig. 1.

The relations between elliptical and Cartesian coordinates are given by

$$
\left\{\begin{array}{l}
x=F \cosh \mu \cos \varphi \\
y=F \sinh \mu \sin \varphi
\end{array}\right.
$$

where $F$ is the focal distance of the ellipse, related to the major and minor semiaxes $a$ and $b$ by

$$
F=\sqrt{a^{2}-b^{2}}
$$

and cosh and sinh are the hyperbolic functions.

With these notations the origin of the coordinate system is given by $(\mu=0, \phi=\pi / 2)$, and the boundary of the beam pipe by

$$
\mu_{b}=\operatorname{arccosh}\left(\frac{a}{F}\right) .
$$

In Ref. [21] we have written, in frequency domain, the longitudinal electric field of a point charge moving with a generic velocity $v=\beta c$, with $c$ the speed of light and $\beta$ the relativistic velocity factor, on the axis of a perfectly conducting elliptic vacuum chamber (monopolar term) as a series of combinations of Mathieu functions of the kind 


$$
\begin{aligned}
E_{z, m}^{0}(\varphi, \mu, q) & \\
= & 2 \pi G \sum_{l=0}^{\infty}(-1)^{l} \frac{A_{0}^{(2 l)}}{p_{2 l}^{\prime}} c e_{2 l}(\varphi,-q) \\
& \times\left[F e k_{2 l}(\mu,-q)-\frac{F e k_{2 l}\left(\mu_{b},-q\right)}{C e_{2 l}\left(\mu_{b},-q\right)} C e_{2 l}(\mu,-q)\right],
\end{aligned}
$$

with

$$
G=j \frac{Z_{0} Q k_{0}}{2 \pi \beta^{2} \gamma^{2}}, \quad q=\left(\frac{k_{0} F}{2 \beta \gamma}\right)^{2}
$$

Here $Q$ is the point charge, $\gamma$ the relativistic energy factor, $Z_{0}$ the vacuum impedance, $k_{0}$ the wave number in free space, equal to $\omega / c$, and we have used the elliptic Mathieu functions described in [24]. In Appendix A we have summarized all the Mathieu functions used throughout the paper and how the expansion coefficients can be determined.

Moreover, in Ref. [22], we have also derived the longitudinal electric field produced by a small dipole oriented along $y$ and $x$, and moving with velocity $\beta c$ as

$$
\begin{aligned}
& E_{z, d_{y}}^{0}(\varphi, \mu, q) \\
& =\pi G_{d} \sum_{l=0}^{\infty}(-1)^{l} \frac{A_{1}^{(2 l+1)}}{p_{2 l+1}^{\prime}} \operatorname{Se}_{2 l+1}(\varphi,-q) \\
& \quad \times\left[G e k_{2 l+1}(\mu,-q)-\frac{G e k_{2 l+1}\left(\mu_{b},-q\right)}{S e_{2 l+1}\left(\mu_{b},-q\right)} S e_{2 l+1}(\mu,-q)\right],
\end{aligned}
$$

$$
\begin{aligned}
& E_{z, d_{x}}^{0}(\varphi, \mu, q) \\
& =\pi G_{d} \sum_{l=0}^{\infty}(-1)^{l} \frac{B_{1}^{(2 l+1)}}{s_{2 l+1}^{\prime}} c e_{2 l+1}(\varphi,-q) \\
& \quad \times\left[F e k_{2 l+1}(\mu,-q)-\frac{F e k_{2 l+1}\left(\mu_{b},-q\right)}{C e_{2 l+1}\left(\mu_{b},-q\right)} C e_{2 l+1}(\mu,-q)\right],
\end{aligned}
$$

with

$$
G_{d}=j Z_{0} \frac{P k_{0}^{2}}{2 \pi \beta^{3} \gamma^{3}}
$$

and the dipole moment $P=2 Q d$ with $2 d$ the distance between the two charges of the dipole.

In the above equations, the first term on the right-hand side gives the direct field, while the second term represents the scattered (or indirect) field due to the elliptic boundary conditions.
Starting from the above expressions of the fields, which are valid for a perfectly conducting vacuum chamber, in the next two sections we derive the fields in presence of a finite conductivity of the walls.

\section{LONGITUDINAL ELECTRIC FIELD OF A POINT CHARGE DUE TO THE FINITE CONDUCTIVITY}

Let us suppose to have a perfectly conducting elliptic beam pipe. The azimuthal transverse magnetic field due to a point charge in an elliptical vacuum chamber can be obtained with the directional derivative perpendicular to $\varphi$ of the longitudinal electric field of Eq. (4), which gives

$H_{\varphi, m}(\varphi, \mu, q)=\frac{\beta^{2} \gamma^{2}}{j k_{0} Z_{0}} \frac{\sqrt{2}}{F \sqrt{\cosh 2 \mu-\cos 2 \varphi}} \frac{\partial E_{z, m}^{0}(\varphi, \mu, q)}{\partial \mu}$.

We now use the hypothesis that the magnetic field does not change in presence of a finite conductivity of the pipe walls, that is, we consider a relative magnetic permeability of about 1. This is a good approximation for copper or aluminium vacuum chambers, even if they have a thin coating of NEG or other material to mitigate electron cloud effects and (or) for pumping purposes [7,8]. As a consequence, we can use Eq. (9) to evaluate the magnetic field also inside the walls. From the knowledge of this field, we can determine the new scattered electric field on the walls due to a finite conductivity $\sigma$, by using the Leontovich condition, which gives

$$
E_{z, m}^{i}\left(\varphi, \mu_{b}, q\right)=Z_{s} H_{\varphi, m}\left(\varphi, \mu_{b}, q\right),
$$

with $Z_{s}$ the surface impedance. The above equation is valid for cases when the radius of curvature of the conducting surface is large with respect to the skin depth $\delta$ defined as

$$
\delta=\sqrt{\frac{2 c}{|\omega| \sigma_{c} Z_{0}}} .
$$

Under this condition, the resulting field inside the wall can be well approximated by plane waves. When we evaluate the above fields in $\mu_{b}$, we obtain that the term of Eq. (4) into square brackets becomes proportional to one of the Wronskians of the Mathieu functions [24], which, by definition are independent on $\mu$, such that we can write

$$
\begin{aligned}
E_{z, m}^{i}\left(\varphi, \mu_{b}, q\right)= & \frac{Q Z_{s} \sqrt{2}}{F} \sum_{l=0}^{\infty} \frac{(-1)^{l} A_{0}^{(2 l)} c e_{2 l}(\varphi,-q)}{p_{2 l}^{\prime} \sqrt{\cosh 2 \mu_{b}-\cos 2 \varphi}} \\
& \times \frac{W_{2 l, m}(-q)}{C e_{2 l}\left(\mu_{b},-q\right)},
\end{aligned}
$$

with the Wronskian that can be written as 


$$
W_{2 l, m}(-q)=-(-1)^{l} \frac{p_{2 l}^{\prime}}{\pi A_{0}^{(2 l)}} c e_{2 l}(0,-q) c e_{2 l}\left(\frac{\pi}{2},-q\right) .
$$

In order to determine this electric field also inside the beam pipe, we suppose that the new total longitudinal electric field is equal to the sum of that of Eq. (4) with perfectly conducting walls, plus a term having the same dependence on $\varphi$ and $\mu$ as the scattered field in Eq. (4), with unknown amplitude due to the finite conductivity. This third term can then be written as

$$
\begin{aligned}
E_{z, m}^{i}(\varphi, \mu, q)= & \frac{Q Z_{s} \sqrt{2}}{F} \sum_{p=0}^{\infty}(-1)^{p} D_{2 p} c e_{2 p}(\varphi,-q) \\
& \times C e_{2 p}(\mu,-q)
\end{aligned}
$$

with $D_{2 p}$ unknown quantities.

The total field, at the elliptic boundary, must be equal to Eq. (12). Since Eq. (4) at $\mu=\mu_{b}$ is zero, we remain with the condition

$$
\begin{aligned}
& \sum_{p=0}^{\infty}(-1)^{p} D_{2 p} c e_{2 p}(\varphi,-q) C e_{2 p}\left(\mu_{b},-q\right) \\
& \quad=\sum_{l=0}^{\infty} \frac{(-1)^{l} A_{0}^{(2 l)} c e_{2 l}(\varphi,-q)}{p_{2 l}^{\prime} \sqrt{\cosh 2 \mu_{b}-\cos 2 \varphi}} \frac{W_{2 l, m}(-q)}{C e_{2 l}\left(\mu_{b},-q\right)}
\end{aligned}
$$

which can be used to determine the unknown expansion coefficients. By using the orthogonality properties of the Mathieu functions, after some manipulations shown in Appendix B, we obtain

$$
\begin{aligned}
D_{2 p}= & \frac{-1}{\pi^{2} C e_{2 p}\left(\mu_{b},-q\right)} \sum_{l=0}^{\infty} \frac{(-1)^{l} c e_{2 l}(0,-q) c e_{2 l}\left(\frac{\pi}{2},-q\right)}{C e_{2 l}\left(\mu_{b},-q\right)} \\
& \times \sum_{r=0}^{\infty} \sum_{t=0}^{\infty}(-1)^{r+t} A_{2 r}^{(2 p)} A_{2 t}^{(2 l)} L_{r, t}^{m}\left(\mu_{b}\right),
\end{aligned}
$$

where

$$
\begin{aligned}
L_{r, t}^{m}\left(\mu_{b}\right)= & \frac{\sqrt{2} \pi e^{-(2|r-t|+1) \mu_{b}} \Gamma\left(\frac{1}{2}+|r-t|\right)}{\Gamma\left(\frac{1}{2}\right)|r-t| !} F\left(\frac{1}{2},|r-t|+\frac{1}{2} ;|r-t|+1 ; e^{-4 \mu_{b}}\right) \\
& +\frac{\sqrt{2} \pi e^{-(2 r+2 t+1) \mu_{b}} \Gamma\left(\frac{1}{2}+r+t\right)}{\Gamma\left(\frac{1}{2}\right)(r+t) !} F\left(\frac{1}{2}, r+t+\frac{1}{2} ; r+t+1 ; e^{-4 \mu_{b}}\right),
\end{aligned}
$$

with $\Gamma$ the gamma function, and $F(a, b ; c ; z)$ the hypergeometric ${ }_{2} F_{1}$ function. It is important to highlight that, thanks to the expansion that we have obtained for $L_{r, t}^{m}$ involving the hypergeometric functions, the summations for the determination of the coefficients $D_{2 p}$ are rapidly convergent and only few terms are necessary to obtain accurate results. The longitudinal electric field due to the finite resistivity of the beam pipe becomes then

$$
\begin{aligned}
E_{z, m}^{i}(\varphi, \mu, q)= & -\frac{Q Z_{s} \sqrt{2}}{\pi^{2} F} \sum_{p=0}^{\infty} \frac{(-1)^{p} c e_{2 p}(\varphi,-q) C e_{2 p}(\mu,-q)}{C e_{2 p}\left(\mu_{b},-q\right)} \\
& \times \sum_{l=0}^{\infty} \frac{(-1)^{l} c e_{2 l}(0,-q) c e_{2 l}\left(\frac{\pi}{2},-q\right)}{C e_{2 l}\left(\mu_{b},-q\right)} \sum_{r=0}^{\infty} \sum_{t=0}^{\infty}(-1)^{r+t} A_{2 r}^{(2 p)} A_{2 t}^{(2 l)} L_{r, t}^{m}\left(\mu_{b}\right) .
\end{aligned}
$$

\section{LONGITUDINAL ELECTRIC FIELD OF A SMALL DIPOLE DUE TO THE FINITE CONDUCTIVITY}

The procedure to determine the longitudinal electric field of a small dipole in case of a finite conductivity of the pipe walls is similar as that of the point charge. We first obtain the azimuthal magnetic field as in Eq. (9) by using the longitudinal electric field of the vertical or horizontal dipole of Eqs. (6) and (7), and then we suppose that the magnetic field does not change in presence of a finite conductivity, so that the new scattered electric field on the walls in the two planes can be written as

$$
E_{z, d_{y}}^{i}\left(\varphi, \mu_{b}, q\right)=\frac{P Z_{s} k_{0} \sqrt{2}}{2 \beta \gamma F} \sum_{l=0}^{\infty} \frac{(-1)^{l} A_{1}^{(2 l+1)} s e_{2 l+1}(\varphi,-q)}{p_{2 l+1}^{\prime} \sqrt{\cosh 2 \mu_{b}-\cos 2 \varphi}} \frac{W_{2 l+1, d_{y}}(-q)}{S e_{2 l+1}\left(\mu_{b},-q\right)},
$$




$$
E_{z, d_{x}}^{i}\left(\varphi, \mu_{b}, q\right)=\frac{P Z_{s} k_{0} \sqrt{2}}{2 \beta \gamma F} \sum_{l=0}^{\infty} \frac{(-1)^{l} B_{1}^{(2 l+1)} c e_{2 l+1}(\varphi,-q)}{s_{2 l+1}^{\prime} \sqrt{\cosh 2 \mu_{b}-\cos 2 \varphi}} \frac{W_{2 l+1, d_{x}}(-q)}{C e_{2 l+1}\left(\mu_{b},-q\right)},
$$

with the two Wronskians equal to

$$
\begin{aligned}
& W_{2 l+1, d_{y}}(-q)=-(-1)^{l} \frac{2 \beta \gamma p_{2 l+1}^{\prime}}{\pi k_{0} A_{1}^{(2 l+1)} F} s e_{2 l+1}^{\prime}(0,-q) s e_{2 l+1}\left(\frac{\pi}{2},-q\right), \\
& W_{2 l+1, d_{x}}(-q)=(-1)^{l} \frac{2 \beta \gamma s_{2 l+1}^{\prime}}{\pi k_{0} B_{1}^{(2 l+1)} F} c e_{2 l+1}(0,-q) c e_{2 l+1}^{\prime}\left(\frac{\pi}{2},-q\right) .
\end{aligned}
$$

We then use, for the scattered field inside the beam pipe, an expression similar to that of Eq. (14), but with azimuthal and radial dependencies as $s e_{2 l+1}(\varphi,-q)$ and $S e_{2 l+1}(\mu,-q)$ for the vertical plane and $c e_{2 l+1}(\varphi,-q)$ and $C e_{2 l+1}(\mu,-q)$ for the horizontal one. By following calculations similar to those of Appendix B, we finally obtain

$$
\begin{aligned}
E_{z, d_{y}}^{i}(\varphi, \mu, q)= & -\frac{P Z_{s} \sqrt{2}}{\pi^{2} F^{2}} \sum_{p=0}^{\infty} \frac{(-1)^{p} s e_{2 p+1}(\varphi,-q) S e_{2 p+1}(\mu,-q)}{S e_{2 p+1}\left(\mu_{b},-q\right)} \\
& \times \sum_{l=0}^{\infty} \frac{(-1)^{l} s e_{2 l+1}^{\prime}(0,-q) s e_{2 l+1}\left(\frac{\pi}{2},-q\right)}{S e_{2 l+1}\left(\mu_{b},-q\right)} \sum_{r=0}^{\infty} \sum_{t=0}^{\infty}(-1)^{r+t} A_{2 r+1}^{(2 p+1)} A_{2 t+1}^{(2 l+1)} L_{r, t}^{d_{y}}\left(\mu_{b}\right), \\
E_{z, d_{x}}^{i}(\varphi, \mu, q)= & \frac{P Z_{s} \sqrt{2}}{\pi^{2} F^{2}} \sum_{p=0}^{\infty} \frac{(-1)^{p} c e_{2 p+1}(\varphi,-q) C e_{2 p+1}(\mu,-q)}{C e_{2 p+1}\left(\mu_{b},-q\right)} \\
& \times \sum_{l=0}^{\infty} \frac{(-1)^{l} c e_{2 l+1}(0,-q) c e_{2 l+1}^{\prime}\left(\frac{\pi}{2},-q\right)}{C e_{2 l+1}\left(\mu_{b},-q\right)} \sum_{r=0}^{\infty} \sum_{t=0}^{\infty}(-1)^{r+t} B_{2 r+1}^{(2 p+1)} B_{2 t+1}^{(2 l+1)} L_{r, t}^{d_{x}}\left(\mu_{b}\right),
\end{aligned}
$$

where

$$
\begin{aligned}
L_{r, t}^{d_{y, x}}\left(\mu_{b}\right)= & \frac{\sqrt{2} \pi e^{-(2|r-t|+1) \mu_{b}} \Gamma\left(\frac{1}{2}+|r-t|\right)}{\Gamma\left(\frac{1}{2}\right)|r-t| !} F\left(\frac{1}{2},|r-t|+\frac{1}{2} ;|r-t|+1 ; e^{-4 \mu_{b}}\right) \\
& \pm \frac{\sqrt{2} \pi e^{-(2 r+2 t+3) \mu_{b}} \Gamma\left(\frac{3}{2}+r+t\right)}{\Gamma\left(\frac{1}{2}\right)(r+t+1) !} F\left(\frac{1}{2}, r+t+\frac{3}{2} ; r+t+2 ; e^{-4 \mu_{b}}\right)
\end{aligned}
$$

with the upper sign for the horizontal plane and the minus for the vertical one.

\section{RESISTIVE WALL IMPEDANCES FOR ELLIPTICAL GEOMETRIES}

From the fields given by Eqs. (18), (23), and (24), it is possible to derive all the coupling impedances. In particular, the longitudinal impedance is

$$
\begin{aligned}
Z_{\|}= & -\frac{E_{z, m}^{i}\left(\varphi=\frac{\pi}{2}, \mu=0, q\right)}{Q}=\frac{Z_{s} \sqrt{2}}{\pi^{2} F} \sum_{p=0}^{\infty}(-1)^{p} \frac{c e_{2 p}\left(\frac{\pi}{2},-q\right) C e_{2 p}(0,-q)}{C e_{2 p}\left(\mu_{b},-q\right)} \\
& \times \sum_{l=0}^{\infty}(-1)^{l} \frac{c e_{2 l}(0,-q) c e_{2 l}\left(\frac{\pi}{2},-q\right)}{C e_{2 l}\left(\mu_{b},-q\right)} \sum_{r=0}^{\infty} \sum_{t=0}^{\infty}(-1)^{r+t} A_{2 r}^{(2 p)} A_{2 t}^{(2 l)} L_{r, t}^{m}\left(\mu_{b}\right),
\end{aligned}
$$

while the transverse driving impedances [25] are 


$$
\begin{aligned}
Z_{\perp, y}^{d}= & -\left.\frac{\beta}{F P k_{0}} \frac{\partial E_{z, d_{y}}^{i}}{\partial \mu}\right|_{\varphi=\frac{\pi}{2}, \mu=0}=\frac{Z_{s} \beta \sqrt{2}}{\pi^{2} k_{0} F^{3}} \sum_{p=0}^{\infty}(-1)^{p} \frac{s e_{2 p+1}\left(\frac{\pi}{2},-q\right) S e_{2 p+1}^{\prime}(0,-q)}{S e_{2 p+1}\left(\mu_{b},-q\right)} \\
& \times \sum_{l=0}^{\infty}(-1)^{l} \frac{s e_{2 l+1}^{\prime}(0,-q) s e_{2 l+1}\left(\frac{\pi}{2},-q\right)}{S e_{2 l+1}\left(\mu_{b},-q\right)} \sum_{r=0}^{\infty} \sum_{t=0}^{\infty}(-1)^{r+t} A_{2 r+1}^{(2 p+1)} A_{2 t+1}^{(2 l+1)} L_{r, t}^{d_{y}}\left(\mu_{b}\right), \\
Z_{\perp, x}^{d}= & \left.\frac{\beta}{F P k_{0}} \frac{\partial E_{z, d_{x}}^{i}}{\partial \varphi}\right|_{\varphi=\frac{\pi}{2}, \mu=0}=\frac{Z_{s} \beta \sqrt{2}}{\pi^{2} k_{0} F^{3}} \sum_{p=0}^{\infty}(-1)^{p} \frac{c e_{2 p+1}^{\prime}\left(\frac{\pi}{2},-q\right) C e_{2 p+1}(0,-q)}{C e_{2 p+1}\left(\mu_{b},-q\right)} \\
& \times \sum_{l=0}^{\infty}(-1)^{l} \frac{c e_{2 l+1}(0,-q) c e_{2 l+1}^{\prime}\left(\frac{\pi}{2},-q\right)}{C e_{2 l+1}\left(\mu_{b},-q\right)} \sum_{r=0}^{\infty} \sum_{t=0}^{\infty}(-1)^{r+t} B_{2 r+1}^{(2 p+1)} B_{2 t+1}^{(2 l+1)} L_{r, t}^{d_{x}}\left(\mu_{b}\right),
\end{aligned}
$$

and the detuning (or quadrupolar) ones are

$$
\begin{aligned}
Z_{\perp, y}^{q}= & -\left.\frac{\beta}{F^{2} Q k_{0}} \frac{\partial^{2} E_{z, m}^{i}}{\partial \mu^{2}}\right|_{\varphi=\frac{\pi}{2}, \mu=0}=\frac{Z_{s} \beta \sqrt{2}}{\pi^{2} k_{0} F^{3}} \sum_{p=0}^{\infty}(-1)^{p} \frac{c e_{2 p}\left(\frac{\pi}{2},-q\right) C e_{2 p}^{\prime \prime}(0,-q)}{C e_{2 p}\left(\mu_{b},-q\right)} \\
& \times \sum_{l=0}^{\infty}(-1)^{l} \frac{c e_{2 l}(0,-q) c e_{2 l}\left(\frac{\pi}{2},-q\right)}{C e_{2 l}\left(\mu_{b},-q\right)} \sum_{r=0}^{\infty} \sum_{t=0}^{\infty}(-1)^{r+t} A_{2 r}^{(2 p)} A_{2 t}^{(2 l)} L_{r, t}^{m}\left(\mu_{b}\right), \\
Z_{\perp, x}^{q}= & -\left.\frac{\beta}{F^{2} Q k_{0}} \frac{\partial^{2} E_{z, m}^{i}}{\partial \varphi^{2}}\right|_{\varphi=\frac{\pi}{2}, \mu=0}=\frac{Z_{s} \beta \sqrt{2}}{\pi^{2} k_{0} F^{3}} \sum_{p=0}^{\infty}(-1)^{p} \frac{c e_{2 p}^{\prime \prime}\left(\frac{\pi}{2},-q\right) C e_{2 p}(0,-q)}{C e_{2 p}\left(\mu_{b},-q\right)} \\
& \times \sum_{l=0}^{\infty}(-1)^{l} \frac{c e_{2 l}(0,-q) c e_{2 l}\left(\frac{\pi}{2},-q\right)}{C e_{2 l}\left(\mu_{b},-q\right)} \sum_{r=0}^{\infty} \sum_{t=0}^{\infty}(-1)^{r+t} A_{2 r}^{(2 p)} A_{2 t}^{(2 l)} L_{r, t}^{m}\left(\mu_{b}\right) .
\end{aligned}
$$

All the above impedances have been written per unit length. They depend on the frequency, on the relativistic parameters $\beta$ and $\gamma$ and on the pipe geometry, that is $a$ and $b$. These impedances can also be used with surface impedances other than that of the resistive wall, as the case of wall roughness, or small corrugations in elliptic vacuum chambers.

It is important to underline that, despite the quadruple infinite sums appearing in the expressions of the impedances, due to the very rapid convergence of the Mathieu functions, only a number of terms in the order of 10-30 can be used for the cases of interest in particle accelerators. When the vacuum chamber tends to be flat, the number of terms must be a bit higher, up to 50. However, even in the worse case, such calculations require a few tens of seconds on a normal PC.

Similar fields and impedances have been obtained from the Bessel functions for the circular geometry with radius $b$. For example for the point charge and a vertical dipole at any energy we have

$$
E_{z, m, \mathrm{circ}}^{i}(r)=-\frac{Q Z_{s}}{2 \pi b I_{0}^{2}\left(\frac{k_{0} b}{\beta \gamma}\right)} I_{0}\left(\frac{k_{0} r}{\beta \gamma}\right)
$$

$$
E_{z, d_{y}, \mathrm{circ}}^{i}(r, \theta)=-\frac{P Z_{s} k_{0}}{2 \pi b \beta \gamma I_{1}^{2}\left(\frac{k_{0} b}{\beta \gamma}\right)} \sin \theta I_{1}\left(\frac{k_{0} r}{\beta \gamma}\right),
$$

from which we derive the impedances [26]

$$
\begin{gathered}
Z_{\|, \text {circ }}=\frac{Z_{s}}{2 \pi b I_{0}^{2}\left(\frac{k_{0} b}{\beta \gamma}\right)}, \\
Z_{\perp, \text { circ }}^{d}=\frac{Z_{s} k_{0}}{4 \pi b \beta \gamma^{2} I_{1}^{2}\left(\frac{k_{0} b}{\beta \gamma}\right)}, \\
Z_{\perp, \text { circ }}^{q}=\frac{Z_{s} k_{0}}{4 \pi b \beta \gamma^{2} I_{0}^{2}\left(\frac{k_{0} b}{\beta \gamma}\right)} .
\end{gathered}
$$

In Appendix $\mathrm{C}$ we show that, in the limit when $a \rightarrow b$, Eqs. (26)-(30) become (33)-(35). It is important to observe that a quadrupolar impedance different from zero exists also in a circular beam pipe and, in case of high energy $(\beta=1)$ and not too high frequency such that the argument of the Bessel functions is much lower than 1 , we obtain the well-known expression 


$$
Z_{\|, \mathrm{circ}}=\frac{Z_{s}}{2 \pi b}=\left(\frac{b^{2} \omega}{2 c}\right) Z_{\perp, \mathrm{circ}}^{d}
$$

while the quadrupolar impedance tends to zero.

\section{THE YOKOYA FORM FACTORS}

In case of an ultrarelativistic beam, the resistive wall impedances for the elliptic geometry can be expressed by means of the Yokoya form factors by using the impedance of a circular pipe [15]. These factors depend only on the coefficient $q_{r}=(a-b) /(a+b)$. We can extend the concept of form factors to any energy by dividing Eq. (26) by (33) and (27)-(30) by (34). We then obtain for all the planes

$$
\begin{aligned}
& f_{\|}=\frac{2 \sqrt{2}}{\pi} \frac{b}{F} I_{0}^{2}\left(\frac{k_{0} b}{\beta \gamma}\right) \sum_{p=0}^{\infty}(-1)^{p} \frac{c e_{2 p}\left(\frac{\pi}{2},-q\right) C e_{2 p}(0,-q)}{C e_{2 p}\left(\mu_{b},-q\right)} \\
& \times \sum_{l=0}^{\infty}(-1)^{l} \frac{c e_{2 l}(0,-q) c e_{2 l}\left(\frac{\pi}{2},-q\right)}{C e_{2 l}\left(\mu_{b},-q\right)} \sum_{r=0}^{\infty} \sum_{t=0}^{\infty}(-1)^{r+t} A_{2 r}^{(2 p)} A_{2 t}^{(2 l)} L_{r, t}^{m}\left(\mu_{b}\right), \\
& f_{\perp, y}^{d}=\frac{4 \sqrt{2}}{\pi} I_{1}^{2}\left(\frac{k_{0} b}{\beta \gamma}\right)\left(\frac{\beta \gamma}{k_{0} b}\right)^{2} \frac{b^{3}}{F^{3}} \sum_{p=0}^{\infty}(-1)^{p} \frac{s e_{2 p+1}\left(\frac{\pi}{2},-q\right) S e_{2 p+1}^{\prime}(0,-q)}{S e_{2 p+1}\left(\mu_{b},-q\right)} \\
& \times \sum_{l=0}^{\infty}(-1)^{l} \frac{s e_{2 l+1}^{\prime}(0,-q) s e_{2 l+1}\left(\frac{\pi}{2},-q\right)}{S e_{2 l+1}\left(\mu_{b},-q\right)} \sum_{r=0}^{\infty} \sum_{t=0}^{\infty}(-1)^{r+t} A_{2 r+1}^{(2 p+1)} A_{2 t+1}^{(2 l+1)} L_{r, t}^{d_{y}}\left(\mu_{b}\right), \\
& f_{\perp, x}^{d}=\frac{4 \sqrt{2}}{\pi} I_{1}^{2}\left(\frac{k_{0} b}{\beta \gamma}\right)\left(\frac{\beta \gamma}{k_{0} b}\right)^{2} \frac{b^{3}}{F^{3}} \sum_{p=0}^{\infty}(-1)^{p} \frac{c e_{2 p+1}^{\prime}\left(\frac{\pi}{2},-q\right) C e_{2 p+1}(0,-q)}{C e_{2 p+1}\left(\mu_{b},-q\right)} \\
& \times \sum_{l=0}^{\infty}(-1)^{l} \frac{c e_{2 l+1}(0,-q) c e_{2 l+1}^{\prime}\left(\frac{\pi}{2},-q\right)}{C e_{2 l+1}\left(\mu_{b},-q\right)} \sum_{r=0}^{\infty} \sum_{t=0}^{\infty}(-1)^{r+t} B_{2 r+1}^{(2 p+1)} B_{2 t+1}^{(2 l+1)} L_{r, t}^{d_{x}}\left(\mu_{b}\right), \\
& f_{\perp, y}^{q}=\frac{4 \sqrt{2}}{\pi} I_{1}^{2}\left(\frac{k_{0} b}{\beta \gamma}\right)\left(\frac{\beta \gamma}{k_{0} b}\right)^{2} \frac{b^{3}}{F^{3}} \sum_{p=0}^{\infty}(-1)^{p} \frac{c e_{2 p}\left(\frac{\pi}{2},-q\right) C e_{2 p}^{\prime \prime}(0,-q)}{C e_{2 p}\left(\mu_{b},-q\right)} \\
& \times \sum_{l=0}^{\infty}(-1)^{l} \frac{c e_{2 l}(0,-q) c e_{2 l}\left(\frac{\pi}{2},-q\right)}{C e_{2 l}\left(\mu_{b},-q\right)} \sum_{r=0}^{\infty} \sum_{t=0}^{\infty}(-1)^{r+t} A_{2 r}^{(2 p)} A_{2 t}^{(2 l)} L_{r, t}^{m}\left(\mu_{b}\right), \\
& f_{\perp, x}^{q}=\frac{4 \sqrt{2}}{\pi} I_{1}^{2}\left(\frac{k_{0} b}{\beta \gamma}\right)\left(\frac{\beta \gamma}{k_{0} b}\right)^{2} \frac{b^{3}}{F^{3}} \sum_{p=0}^{\infty}(-1)^{p} \frac{c e_{2 p}^{\prime \prime}\left(\frac{\pi}{2},-q\right) C e_{2 p}(0,-q)}{C e_{2 p}\left(\mu_{b},-q\right)} \\
& \times \sum_{l=0}^{\infty}(-1)^{l} \frac{c e_{2 l}(0,-q) c e_{2 l}\left(\frac{\pi}{2},-q\right)}{C e_{2 l}\left(\mu_{b},-q\right)} \sum_{r=0}^{\infty} \sum_{t=0}^{\infty}(-1)^{r+t} A_{2 r}^{(2 p)} A_{2 t}^{(2 l)} L_{r, t}^{m}\left(\mu_{b}\right) .
\end{aligned}
$$

These form factors depend on the geometry and on the parameter $k_{0} / \beta \gamma$. They are shown in Fig. 2 as a function of $q_{r}$ for different values of the argument of the Bessel function $k_{p}=k_{0} b / \beta \gamma$. We can observe that for a fixed beam velocity and geometry (moving along the vertical axis), they also change with frequency.

Moreover, the blue curve in all plots $\left(k_{p}=10^{-4}\right)$ corresponds to an ultrarelativistic beam. In this case, that is when $q \rightarrow 0$, it is possible to simplify the form factors showing that they do not depend on the frequency any more. Indeed, as already discussed in Appendix C, all the coefficients of the Mathieu expansions are negligible except the diagonal ones, which are all equal to unity except $A_{0}^{(0)}=1 / \sqrt{2}$. Therefore we can keep only the first order expansion of the Mathieu functions which does not depend on $q$. Moreover the last two summations of the above form factors have only the terms $r=p$ and $t=l$. We can then finally write 

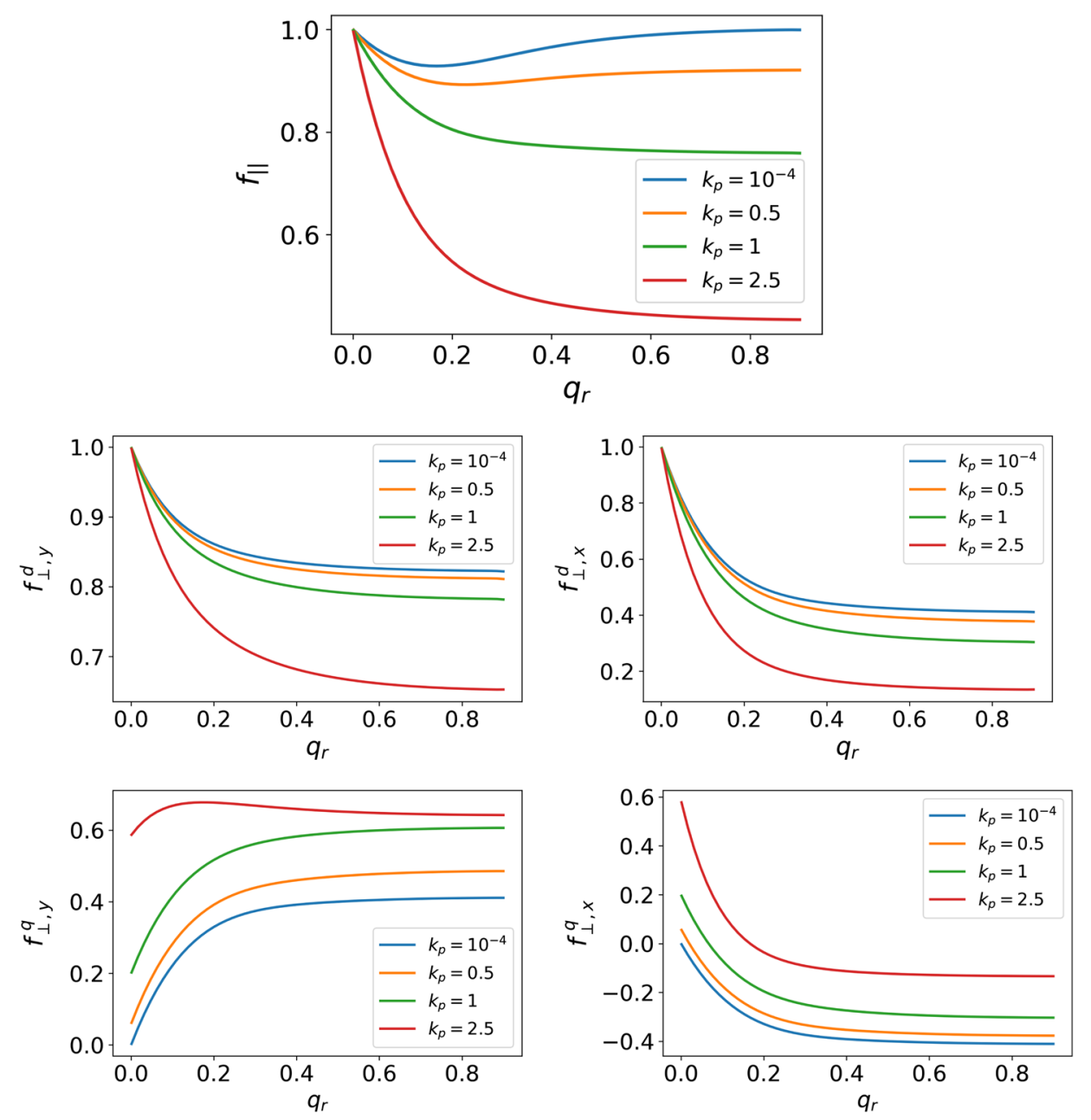

FIG. 2. Form factors as a function of $q_{r}=(a-b) /(a+b)$ for different values of $k_{p}=k_{0} b /(\beta \gamma)$.

$$
\begin{gathered}
f_{\|}(\gamma \rightarrow \infty)=\frac{2 \sqrt{2}}{\pi} \frac{b}{F} \sum_{p=0}^{\infty}(-1)^{p} \frac{1}{\epsilon_{0, p} \cosh \left(2 p \mu_{b}\right)} \sum_{l=0}^{\infty}(-1)^{l} \frac{1}{\epsilon_{0, l} \cosh \left(2 l \mu_{b}\right)} L_{p, l}^{m}\left(\mu_{b}\right), \\
f_{\perp, y}^{d}(\gamma \rightarrow \infty)=\frac{\sqrt{2}}{\pi} \frac{b^{3}}{F^{3}} \sum_{p=0}^{\infty}(-1)^{p} \frac{2 p+1}{\sinh \left[(2 p+1) \mu_{b}\right]} \sum_{l=0}^{\infty}(-1)^{l} \frac{2 l+1}{\sinh \left[(2 l+1) \mu_{b}\right]} L_{p, l}^{d_{y}}\left(\mu_{b}\right), \\
f_{\perp, x}^{d}(\gamma \rightarrow \infty)=\frac{\sqrt{2}}{\pi} \frac{b^{3}}{F^{3}} \sum_{p=0}^{\infty}(-1)^{p} \frac{2 p+1}{\cosh \left[(2 p+1) \mu_{b}\right.} \sum_{l=0}^{\infty}(-1)^{l} \frac{2 l+1}{\cosh \left[(2 l+1) \mu_{b}\right.} L_{p, l}^{d_{x}}\left(\mu_{b}\right), \\
f_{\perp, y, x}^{q}(\gamma \rightarrow \infty)= \pm \frac{\sqrt{2}}{\pi} \frac{b^{3}}{F^{3}} \sum_{p=0}^{\infty}(-1)^{p} \frac{(2 p)^{2}}{\cosh \left(2 p \mu_{b}\right)} \sum_{l=0}^{\infty}(-1)^{l} \frac{1}{\epsilon_{0, l} \cosh \left(2 l \mu_{b}\right)} L_{p, l}^{m}\left(\mu_{b}\right),
\end{gathered}
$$

with $\epsilon_{0, p}=1+\delta_{0, p}$ with $\delta_{0, p}$ the Kroneker delta and, for the quadrupolar form factors, the upper sign is for the vertical plane. Equations. (42)-(45) constitute an alternative way to express the Yokoya form factors. Indeed, in Fig. 3 we have compared these expressions with the longitudinal and dipolar form factors written in terms of integrals as described in Ref. [1]. This same figure can also be compared with Fig. 8 of Ref. [15].

As we expect, the worst case for the driving impedances is represented by the circular geometry, for which the form factor is always 1 . However, for a given ellipticity, in particular at low energy, the coupling impedances can be 


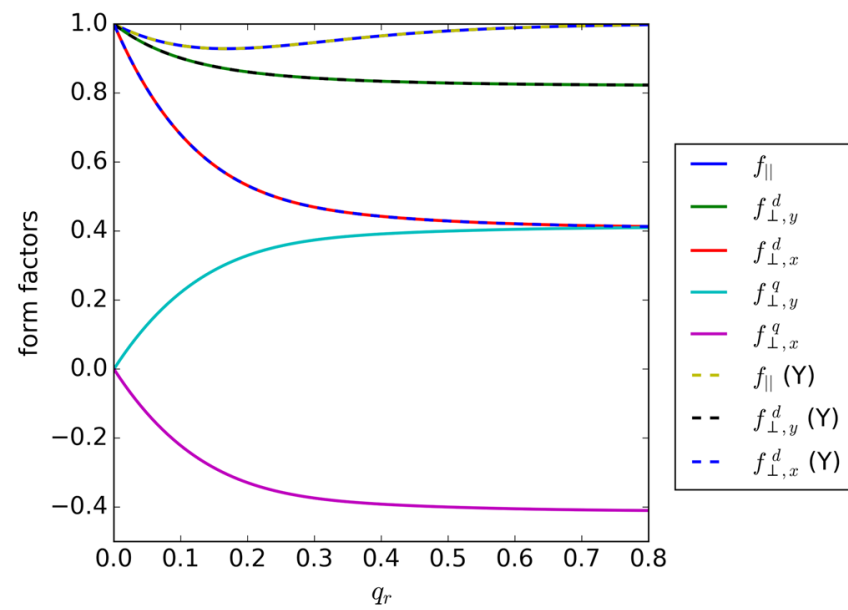

FIG. 3. Yokoya form factors as a function of $q_{r}=(a-b) /$ $(a+b)$. With (Y) we indicate the form factors as expressed by the integrals in Ref. [1].

much lower than those evaluated with the Yokoya form factors. This is also valid for the longitudinal impedance. The contrary happens for the detuning ones, which are zero for the circular geometry in the relativistic case. One interesting result is that the horizontal detuning form factor changes sign passing from positive to negatives values. This happens at a fixed geometry and energy as a function of frequency since $k_{p}$ depends also on this quantity (on the plots of Fig. 2 we move along a vertical line). This is due to the fact that the horizontal detuning impedance for the circular geometry, with $\beta<1$, is equal to the vertical one and they are both positive, while for the flat geometry it is negative. Then, with a given ellipticity, there must be a transition at a given frequency where the impedance passes from positive to negative values.

\section{RESISTIVE WALL IMPEDANCES FOR SIMPLE CASES}

In some simple conditions, it is possible to write an analytical expression of the surface impedance. For example, in the case of a single layer of infinite thickness, for a DC model and in the frequency range [4]

$$
\frac{\chi c}{b} \ll \omega \ll \frac{c \chi^{-1 / 3}}{b},
$$

where $\chi=1 /\left(Z_{0} \sigma_{c} b\right)$ with $\sigma_{c}$ the conductivity of the beam pipe, the surface impedance due to the finite resistivity of the walls can be written as [28]

$$
Z_{s}=[1+j \operatorname{sgn}(\omega)] \sqrt{\frac{|\omega| Z_{0}}{2 \sigma_{c} c}} .
$$

This expression, in combination with Eqs. (26)-(30), allows us to obtain the single layer resistive wall impedances for any ellipticity.

Another interesting application is the case of a beam pipe with a thin layer of coating to reduce the secondary electron yield for electron cloud mitigation and, in some cases, to help the pumping process [29]. As discussed in Ref. [7], under the condition of a good conductor with $\sigma_{c} \gg \omega \varepsilon$ with $\varepsilon$ the material dielectric constant, and with $\omega b /(\gamma \beta c) \ll 1$, the surface impedance can be written as

$Z_{s}=[1+j \operatorname{sgn}(\omega)] \sqrt{\frac{|\omega| Z_{0}}{2 \sigma_{c} c}} \frac{\alpha \tanh \left[\frac{1+j \operatorname{sgn}(\omega)}{\delta_{1}} \Delta\right]+1}{\alpha+\tanh \left[\frac{1+j \operatorname{sgn}(\omega)}{\delta_{1}} \Delta\right]}$,

where $\delta_{1}$ is the skin depth of the coating, $\Delta$ its corresponding thickness, and, for a good conductor, $\alpha \simeq \delta_{1} / \delta_{2}$, with $\delta_{2}$ the skin depth of the substrate, which is supposed to be of infinite thickness. Again, this surface impedance, in combination with Eqs. (26)-(30), allows to obtain the resistive wall impedance for an elliptical beam pipe with a thin coating. When $\Delta \rightarrow 0$ and $\delta_{1}=\delta_{2}$ we obtain the simple case of single layer with infinite thickness.

\section{RESISTIVE WALL IMPEDANCE FOR A MULTILAYER BEAM PIPE}

The surface impedance concept can be extended to the case of beam pipe with multi-layer structure of the wall by using the transmission line (TL) theory. TLWALL [30] is a code developed at CERN and based on the TL theory that, resuming the studies of L. Vos [31-33], allows computing the resistive wall impedance of round chambers.

In spite of its simplicity, the TL theory with some modification can be used to model the resistive wall impedance of multilayer structures without loss of generality. The theory can be applied to calculate the surface impedance of a round chamber loaded by a structure made of an arbitrary number of layers of finite thickness, allowing for PEC, vacuum or conductive wall (lossy materials, i.e., finite electrical conductivity and/or with complex permeability/permittivity) boundary conditions.

In order to evaluate the coupling impedance for an elliptic geometry with a multilayer structure, we use the equivalent surface impedance $Z_{s}$ computed by TLWALL in combination with the fields of the previous sections.

The impedance obtained from TLWALL also includes the inductive bypass effect introduced by Vos [32,34] and the attenuation due to propagation of cylindrical wave introduced in [30] to overcome the limitations of the transmission line approach when the penetration depth becomes comparable to the pipe radius $b$.

The application of the method requires the validity of the Leontovich condition. Moreover, the attenuation due to propagation of cylindrical waves and the inductive bypass concept have been derived for round chambers and are then 
not exact for elliptical chambers. The theory of TLWALL and all the validity limits, which obviously extend to our approach, are discussed in pages 86 to 89 of Ref. [30].

TLWALL has been shown to give very accurate results in a large frequency range (from $\mathrm{kHz}$ to $\mathrm{GHz}$ range) in several cases of interest: metal with vacuum boundary, metal with material (magnetic) boundary, metal with PEC boundary, metal coatings, ceramic insert, ceramic coatings, metamaterials $[30,35,36]$.

\section{BENCHMARK EXAMPLES AND APPLICATIONS}

In this section we show some examples by comparing the results of Eqs. (26)-(30), in combination with the surface impedance of TLWALL for a multilayer structure, with the 3D electromagnetic simulation software CST [37] and IW2D [27], a 2D code developed at CERN for the evaluation of the wall impedance (resistive wall plus space charge) in circular and flat multilayer geometries.

As a first example we evaluate the impedance of a twolayer elliptic beam pipe. The vacuum chamber that we have considered consisted in a first layer with a small conductivity $(\sigma=400 \mathrm{~S} / \mathrm{m})$ with a thickness of $5 \mathrm{~mm}$ followed by a perfect electric conductor. One reason for the small conductivity is due to the fact that we want to compare the results with CST and we need an impedance per meter of structure well above the numerical noise level. In addition to this, CST has two ways of dealing with the resistive wall impedance: if a material is defined "lossy metal," then CST uses the definition of surface impedance similarly to our case; if we define instead the material as "normal," then CST evaluates the fields inside of it in order to determine the coupling impedance, and this is the method we want to use to check our results. Since a small conductivity gives quite a large skin depth, this, associated to the thickness that we
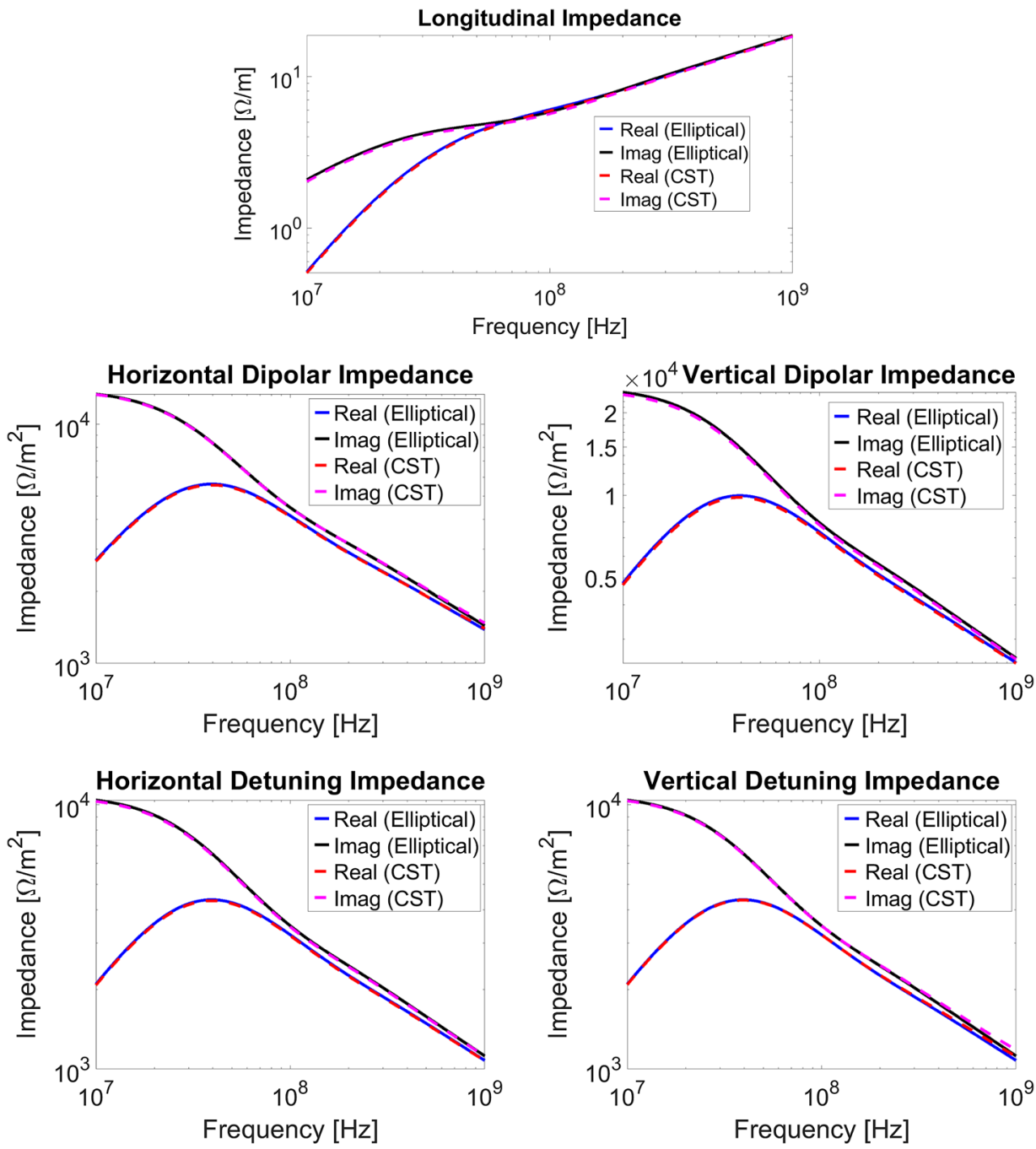

FIG. 4. Coupling impedances per unit of length as given by Eqs. (26)-(30) compared to the results of CST. The beam pipe has a first layer with $\sigma=400 \mathrm{~S} / \mathrm{m}$ and thickness of $5 \mathrm{~mm}$, followed by a perfect electric conductor. The relativistic factor is $\gamma=27.7$ and $q_{r}=0.29$. 
have used, allows to evaluate the fields inside the material with a high number of mesh cells, but not excessive.

For the geometrical parameters, we considered a minor semiaxis $b=25 \mathrm{~mm}$ and a major semiaxis $a=45 \mathrm{~mm}$ $\left(q_{r}=0.29\right)$. These values, as well as $\gamma=27.7$ are representative of the CERN Super Proton Synchrotron vacuum chamber. It is worth mentioning that each simulation is optimized for one frequency decade by using the scaling technique described at page 96 of Ref. [30]. Since the simulations are quite heavy due to the large number of mesh cells required to have a dense mesh in the penetration depth, only two frequency decades have been investigated. The simulations have been performed between $10 \mathrm{MHz}$ and $1 \mathrm{GHz}$. The choice of the decades is such that all the three resistive wall regimes (thin wall, intermediate, and thick wall) are covered.

In Fig. 4 we show the results of Eqs. (26)-(30) compared to CST simulations. The curves have been obtained with 50 terms to get the expansion coefficients $A$ and $B$, and with 30 terms in all the summations. The impedances are given per unit of length. The agreement is very good for all the impedances in the frequency range that we have chosen.

Since with CST we have considered the electromagnetic propagation of the fields inside the materials, Fig. 4, demonstrates that it is possible to combine the fields of the elliptic geometry with the results of TLWALL even in a multilayer system and not only in the case of a single layer.

In Fig. 5 we also show a comparison of the horizontal detuning impedance at quite low beta $(0.52)$ with IW2D in the limits of circular and flat geometries. The radius of the vacuum chamber is $31.3 \mathrm{~mm}$ (corresponding to half

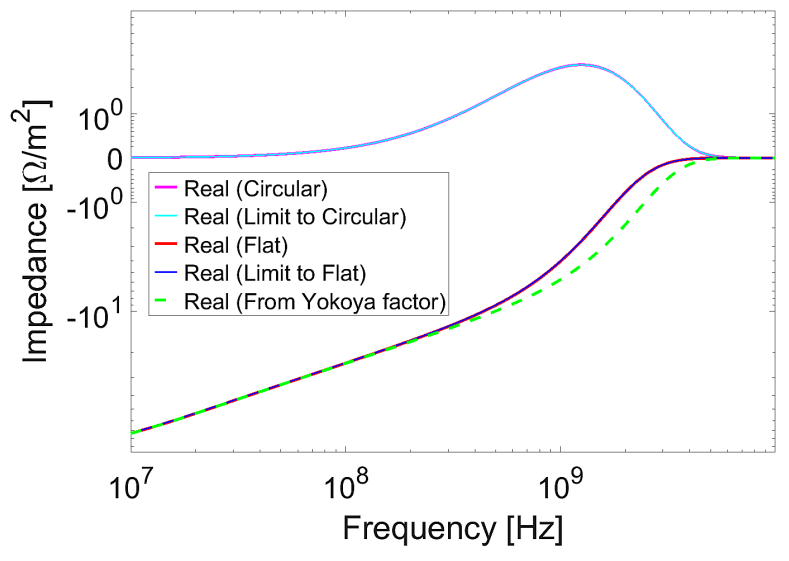

FIG. 5. Real part of the horizontal detuning impedance per unit of length as given by Eq. (30) in the limits of circular and flat geometries (cyan and blue lines, respectively) for a nonrelativistic case $(\beta=0.52)$, compared with IW2D (magenta and red lines) and with a circular pipe multiplied by the Yokoya factor (green dashed line). The beam pipe, with a radius of $31.3 \mathrm{~mm}$ (corresponding to half aperture for the flat beam pipe), has three layers of thicknesses $0.4 \mathrm{~mm}, 3 \mathrm{~mm}$, infinity and respective conductivities of $7.7 \times 10^{5} \mathrm{~S} / \mathrm{m}, 1 \times 10^{4} \mathrm{~S} / \mathrm{m}$, and zero (vacuum boundary). aperture for the flat beam pipe), and we have used three layers of thicknesses $0.4 \mathrm{~mm}, 3 \mathrm{~mm}$, infinity and respective conductivities of $7.7 \times 10^{5} \mathrm{~S} / \mathrm{m}, 1 \times 10^{4} \mathrm{~S} / \mathrm{m}$, and zero (vacuum boundary). These values are very close to those of the CERN PS Booster vacuum chamber. As can be seen from the figure, the results of Eq. (30) for circular and flat chambers (cyan and blue curves, respectively) agree very well with IW2D (magenta and red curves). Moreover it is shown that, with these parameters, by using the Yokoya form factor, some discrepancies appear at frequencies around $1 \mathrm{GHz}$ (also note that the vertical scale is logarithmic).

Of course, with our method we can also cover all the intermediate cases of elliptic geometries that cannot be obtained with IW2D. Indeed, in some conditions, it is not always correct to multiply the impedance of a circular multilayer pipe by the Yokoya form factor to obtain the corresponding elliptic impedance. This is particularly evident if we consider the horizontal detuning impedance, which is zero or positive for a circular pipe and negative for a flat chamber. In all the intermediate cases it is not possible to predict at which frequency the impedance changes sign, and the simple Yokoya form factor (which is negative) times the dipolar impedance of the circular chamber can give, in some cases, incorrect results.

This is clearly shown in Fig. 6, where the real part of the horizontal detuning impedance given by Eq. (30) with $q_{r}=0.1$ and the same other parameters of Fig. 5, in blue, is compared with IW2D (green line) for a circular pipe multiplied by the Yokoya form factor. In a frequency range around $1 \mathrm{GHz}$, the discrepancy, as expected, is quite large, with the correct impedance passing from negative to positive values. This behavior is also confirmed by more tricky and time consuming CST simulations, shown in the

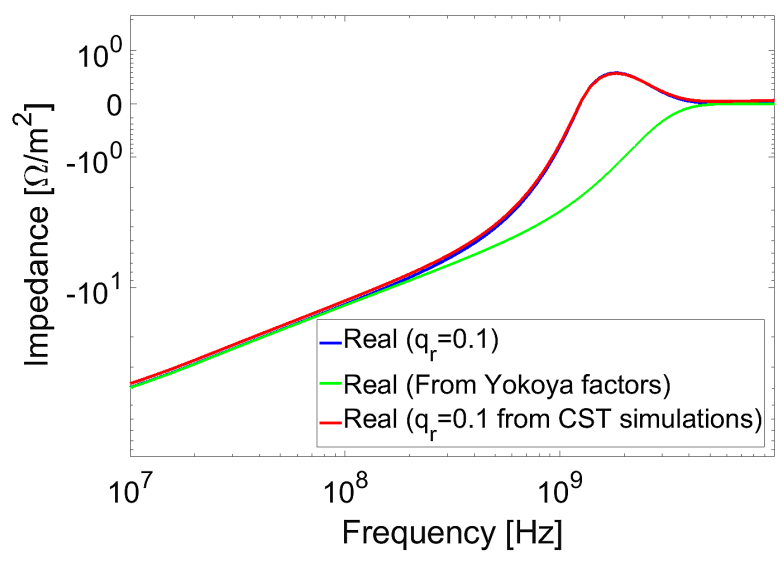

FIG. 6. Real part of the horizontal detuning impedance per unit of length as given by Eq. (30) for $q_{r}=0.1$ (blue curve), compared with IW2D by using the Yokoya factor (green curve). The beam pipe has three layers of thicknesses $0.4 \mathrm{~mm}, 3 \mathrm{~mm}$, infinity and respective conductivities of $7.7 \times 10^{5} \mathrm{~S} / \mathrm{m}$, $1 \times 10^{4} \mathrm{~S} / \mathrm{m}$, and zero (vacuum boundary). 
same figure with the red line [38]. Due to the complexity of the simulations the materials have been modeled using the CST surface impedance features.

\section{CONCLUSIONS}

In this paper we have derived the coupling impedances due to the finite conductivity of an elliptical vacuum chamber. The developed theory can be used to obtain the resistive wall impedance in elliptic geometry by using the concept of surface impedance in combination with the expansions of the electric fields in terms of Mathieu functions.

We have also derived the Yokoya form factors, generalizing them also for the nonrelativistic case and for any frequency range. In this last case, however, the Leontovich condition and the surface impedance concept must be valid.

Moreover, with the use of the theory of transmission lines, we have obtained the resistive wall impedance for a multilayer vacuum chamber with elliptical cross section.

The comparisons of the results with the electromagnetic code CST showed a very good agreement for both ultrarelativistic and low beta cases in a very large frequency range for multi-layer vacuum chambers with any ellipticity. We have also compared the results in the extreme cases of circular and flat geometries with the code IW2D.

The fields that we have obtained in this paper can also be used for a more rigorous method which takes into account the matching conditions between different layers. In this way we can develop a code able to evaluate the wall impedance, including also the resistive wall term, for multilayers vacuum chambers of elliptic cross section without recurring to the Leontovich condition and the concept of surface impedance, extending the code IW2D, currently developed only for the circular case and parallel plates.

\section{ACKNOWLEDGMENTS}

We acknowledge many helpful and stimulating discussions with E. Métral. This work was partially supported by the European Commission under the HORIZON 2020 Integrating Activity project ARIES, Grant agreement No. 730871, by the CERN PS-LIU project, and by INFN National committee $\mathrm{V}$ trough the MICA project.

\section{APPENDIX A: MATHIEU FUNCTIONS AND EXPANSION COEFFICIENTS}

In this Appendix we summarize all the Mathieu functions used in the paper, considering, as reference work, the book of McLachlan [24].

The periodic angular ordinary Mathieu functions are given by four series of orthogonal equations, which are called the elliptic cosine even, cosine odd, sine even, and sine odd functions. For the electric field generated by a point charge and a dipole in an elliptic geometry, we need only the first two and the last one with negative argument $-q$, expressed by:

$$
\begin{gathered}
c e_{2 l}(\varphi,-q)=(-1)^{l} \sum_{r=0}^{\infty}(-1)^{r} A_{2 r}^{(2 l)} \cos (2 r \varphi) \\
c e_{2 l+1}(\varphi,-q)=(-1)^{l} \sum_{r=0}^{\infty}(-1)^{r} B_{2 r+1}^{(2 l+1)} \cos [(2 r+1) \varphi] \\
\operatorname{se}_{2 l+1}(\varphi,-q)=(-1)^{l} \sum_{r=0}^{\infty}(-1)^{r} A_{2 r+1}^{(2 l+1)} \sin [(2 r+1) \varphi],
\end{gathered}
$$

and the corresponding radial modified Mathieu functions of the first and second kind given respectively by

$$
\begin{aligned}
C e_{2 l}(\mu,-q)= & \frac{p_{2 l}^{\prime}}{A_{0}^{(2 l)}} \sum_{r=0}^{\infty}(-1)^{r} A_{2 r}^{(2 l)} I_{r}\left(\nu_{1}\right) I_{r}\left(\nu_{2}\right) \\
C e_{2 l+1}(\mu,-q)= & \frac{s_{2 l+1}^{\prime}}{B_{1}^{(2 l+1)}} \sum_{r=0}^{\infty}(-1)^{r} B_{2 r+1}^{(2 l+1)}\left[I_{r}\left(\nu_{1}\right) I_{r+1}\left(\nu_{2}\right)\right. \\
& \left.+I_{r+1}\left(\nu_{1}\right) I_{r}\left(\nu_{2}\right)\right] \\
\operatorname{Se}_{2 l+1}(\mu,-q)= & \frac{p_{2 l+1}^{\prime}}{A_{1}^{(2 l+1)}} \sum_{r=0}^{\infty}(-1)^{r} A_{2 r+1}^{(2 l+1)}\left[I_{r}\left(\nu_{1}\right) I_{r+1}\left(\nu_{2}\right)\right. \\
& \left.-I_{r+1}\left(\nu_{1}\right) I_{r}\left(\nu_{2}\right)\right],
\end{aligned}
$$

and

$$
F e k_{2 l}(\mu,-q)=\frac{p_{2 l}^{\prime}}{\pi A_{0}^{(2 l)}} \sum_{r=0}^{\infty} A_{2 r}^{(2 l)} I_{r}\left(\nu_{1}\right) K_{r}\left(\nu_{2}\right)
$$

$$
\begin{aligned}
F e k_{2 l+1}(\mu,-q)= & \frac{s_{2 l+1}^{\prime}}{\pi B_{1}^{(2 l+1)}} \sum_{r=0}^{\infty} B_{2 r+1}^{(2 l+1)}\left[I_{r}\left(\nu_{1}\right) K_{r+1}\left(\nu_{2}\right)\right. \\
& \left.-I_{r+1}\left(\nu_{1}\right) K_{r}\left(\nu_{2}\right)\right] \\
\operatorname{Gek}_{2 l+1}(\mu,-q)= & \frac{p_{2 l+1}^{\prime}}{\pi A_{1}^{(2 l+1)}} \sum_{r=0}^{\infty} A_{2 r+1}^{(2 l+1)}\left[I_{r}\left(\nu_{1}\right) K_{r+1}\left(\nu_{2}\right)\right. \\
& \left.+I_{r+1}\left(\nu_{1}\right) K_{r}\left(\nu_{2}\right)\right],
\end{aligned}
$$

with

$$
\begin{array}{r}
p_{2 l}^{\prime}=(-1)^{l} \frac{c e_{2 l}(0,-q) c e_{2 l}\left(\frac{\pi}{2},-q\right)}{A_{0}^{(2 l)}} \\
s_{2 l+1}^{\prime}=(-1)^{l+1} \frac{c e^{\prime}{ }_{2 l+1}\left(\frac{\pi}{2},-q\right) c e_{2 l+1}(0,-q)}{\sqrt{q} B_{1}^{(2 l+1)}}
\end{array}
$$




$$
p_{2 l+1}^{\prime}=(-1)^{l} \frac{s e_{2 l+1}\left(\frac{\pi}{2},-q\right) s e_{2 l+1}^{\prime}(0,-q)}{\sqrt{q} A_{1}^{(2 l+1)}},
$$

and $\nu_{1}=\sqrt{q} e^{-\mu}$ and $\nu_{2}=\sqrt{q} e^{\mu}$. Here $I_{r}(x)$ and $K_{r}(x)$ are the modified Bessel functions of first and second kind, and $s e_{2 l+1}^{\prime}$ and $c e_{2 l+1}^{\prime}$ are the derivatives of the respective functions.

It is important to observe that the radial modified Mathieu functions of the second kind $F e k(\mu,-q)$ and $\operatorname{Gek}(\mu,-q)$ play the role, in elliptic geometry, of what the modified Bessel function of second kind $K(x)$ represent in cylindrical one.

The expansion coefficients $A_{2 r}^{(2 l)}, A_{2 r+1}^{(2 l+1)}$, and $B_{2 r+1}^{(2 l+1)}$, are defined in such a way that the angular functions are orthogonal [24].

They can then be obtained by solving an eigenvalue problem for the following truncated matrices:

$$
\begin{aligned}
a A_{0}^{(2 l)}-q A_{2}^{(2 l)} & =0 \\
{[a-4] A_{2}^{(2 l)}-q\left(2 A_{0}^{(2 l)}+A_{4}^{(2 l)}\right) } & =0 \\
{\left[a-(2 r)^{2}\right] A_{2 r}^{(2 l)}-q\left(A_{2 r-2}^{(2 l)}+A_{2 r+2}^{(2 l)}\right) } & =0 \quad(r \geq 2)
\end{aligned}
$$

$$
\begin{aligned}
(a-1+q) B_{1}^{(2 l+1)}-q B_{3}^{(2 l+1)} & =0 \\
{\left[a-(2 r+1)^{2}\right] B_{2 r+1}^{(2 l+1)}-q\left(B_{2 r-1}^{(2 l+1)}+B_{2 r+3}^{(2 l+1)}\right) } & =0 \quad(r \geq 1)
\end{aligned}
$$

$$
\begin{aligned}
(a-1-q) A_{1}^{(2 l+1)}-q A_{3}^{(2 l+1)} & =0 \\
{\left[a-(2 r+1)^{2}\right] A_{2 r+1}^{(2 l+1)}-q\left(A_{2 r-1}^{(2 l+1)}+A_{2 r+3}^{(2 l+1)}\right) } & =0 \quad(r \geq 1),
\end{aligned}
$$

where the terms " $a$ " represent the eigenvalues and the expansion coefficients are the eigenvectors of the three truncated linear equations' systems.

\section{APPENDIX B: EXPANSION COEFFICIENTS OF THE SCATTERED LONGITUDINAL ELECTRIC FIELD OF A POINT CHARGE WITH CONDUCTIVE WALLS}

If we multiply both sides of Eq. (15) by $(-1)^{m} c e_{2 m}(\varphi,-q)$ and integrate in $\varphi$ from $-\pi$ to $\pi$, by using the orthogonality properties of the Mathieu functions, we obtain

$$
\begin{aligned}
\pi D_{2 m} C e_{2 m}\left(\mu_{b},-q\right) & =\sum_{l=0}^{\infty} \frac{(-1)^{(l+m)} A_{0}^{(2 l)} W_{2 l, m}(-q)}{p_{2 l}^{\prime} C e_{2 l}\left(\mu_{b},-q\right)} \int_{-\pi}^{\pi} \frac{c e_{2 l}(\varphi,-q) c e_{2 m}(\varphi,-q)}{\sqrt{\cosh 2 \mu_{b}-\cos 2 \varphi}} d \varphi \\
& =\sum_{l=0}^{\infty} \frac{A_{0}^{(2 l)} W_{2 l, m}(-q)}{p_{2 l}^{\prime} C e_{2 l}\left(\mu_{b},-q\right)} \sum_{r=0}^{\infty} \sum_{t=0}^{\infty}(-1)^{r+t} A_{2 r}^{(2 m)} A_{2 t}^{(2 l)} \int_{-\pi}^{\pi} \frac{\cos (2 r \varphi) \cos (2 t \varphi)}{\sqrt{\cosh 2 \mu_{b}-\cos 2 \varphi}} d \varphi
\end{aligned}
$$

The last integral can be written as

$$
\int_{-\pi}^{\pi} \frac{\cos (2 r \varphi) \cos (2 t \varphi)}{\sqrt{\cosh 2 \mu_{b}-\cos 2 \varphi}} d \varphi=\frac{1}{2} \int_{-\pi}^{\pi} \frac{\cos [(2 r-2 t) \varphi]+\cos [(2 r+2 t) \varphi]}{\sqrt{\cosh 2 \mu_{b}-\cos 2 \varphi}} d \varphi .
$$

If we define $p=|r-t|$, the first integral on the right-hand side can be written in terms of the gamma function $\Gamma$, and the hypergeometric ${ }_{2} F_{1}$ function $F(a, b ; c ; z)[39]$ as

$$
\begin{aligned}
\frac{1}{2} \int_{-\pi}^{\pi} \frac{\cos (2 p \varphi)}{\sqrt{\cosh 2 \mu_{b}-\cos 2 \varphi}} d \varphi & =\frac{e^{-\mu_{b}}}{\sqrt{2}} \int_{0}^{2 \pi} \frac{\cos (p s)}{\sqrt{1+e^{-4 \mu_{b}}-2 e^{-2 \mu_{b}} \cos s}} d s \\
& =\frac{\sqrt{2} \pi e^{-(2 p+1) \mu_{b}} \Gamma\left(\frac{1}{2}+p\right)}{\Gamma\left(\frac{1}{2}\right) p !} F\left(\frac{1}{2}, p+\frac{1}{2} ; p+1 ; e^{-4 \mu_{b}}\right) .
\end{aligned}
$$

A similar expression can be found for the second integral containing $(2 r+2 t)$, such that we find finally

$$
\begin{aligned}
\int_{-\pi}^{\pi} \frac{\cos (2 r \varphi) \cos (2 t \varphi)}{\sqrt{\cosh 2 \mu_{b}-\cos 2 \varphi}} d \varphi= & \frac{\sqrt{2} \pi e^{-(2|r-t|+1) \mu_{b}} \Gamma\left(\frac{1}{2}+|r-t|\right)}{\Gamma\left(\frac{1}{2}\right)|r-t| !} F\left(\frac{1}{2},|r-t|+\frac{1}{2} ;|r-t|+1 ; e^{-4 \mu_{b}}\right) \\
& +\frac{\sqrt{2} \pi e^{-(2 r+2 t+1) \mu_{b}} \Gamma\left(\frac{1}{2}+r+t\right)}{\Gamma\left(\frac{1}{2}\right)(r+t) !} F\left(\frac{1}{2}, r+t+\frac{1}{2} ; r+t+1 ; e^{-4 \mu_{b}}\right),
\end{aligned}
$$

which, substituted in Eq. (B1), gives, for $D_{2 m}$ the expression of Eq. (16). 


\section{APPENDIX C: THE LIMIT OF CIRCULAR PIPE}

In the limit of $a \rightarrow b$, we have that $F \rightarrow 0$ and $q \rightarrow 0$. In these conditions it is possible to expand the Mathieu functions to first order in $q$. All the coefficients of the expansions are negligible except the diagonal ones [24] so that the last two summations in Eqs. (26)-(30) have only $r=p$ and $t=l$. Moreover, since $\mu_{b} \rightarrow \infty$, the radial Mathieu functions $\mathrm{Ce}$ and $\mathrm{Se}$ in the denominators of the equations must be evaluated at very large values. The asymptotic expansions in this case give [24]

$$
\begin{array}{r}
C e_{2 l}\left(\mu_{b} \rightarrow \infty,-q \rightarrow 0\right)=p_{2 l}^{\prime} I_{2 l}\left(\frac{k_{0} b}{\beta \gamma}\right), \\
C e_{2 l+1}\left(\mu_{b} \rightarrow \infty,-q \rightarrow 0\right)=s_{2 l+1}^{\prime} I_{2 l+1}\left(\frac{k_{0} b}{\beta \gamma}\right), \\
S e_{2 l+1}\left(\mu_{b} \rightarrow \infty,-q \rightarrow 0\right)=p_{2 l+1}^{\prime} I_{2 l+1}\left(\frac{k_{0} b}{\beta \gamma}\right),
\end{array}
$$

so that Eqs. (26)-(30) become

$$
\begin{gathered}
Z_{\|}=\frac{Z_{s} \sqrt{2}}{\pi^{2} F} \sum_{p=0}^{\infty} A_{2 p}^{(2 p)} \frac{c e_{2 p}\left(\frac{\pi}{2},-q\right) C e_{2 p}(0,-q)}{p_{2 p}^{\prime} I_{2 p}\left(\frac{k_{0} b}{\beta \gamma}\right)} \sum_{l=0}^{\infty} A_{2 l}^{(2 l)} \frac{c e_{2 l}(0,-q) c e_{2 l}\left(\frac{\pi}{2},-q\right)}{p_{2 l}^{\prime} I_{2 l}\left(\frac{k_{0} b}{\beta \gamma}\right)} L_{p, l}^{m}\left(\mu_{b} \rightarrow \infty\right), \\
Z_{\perp, y}^{d}=\frac{Z_{s} \beta \sqrt{2}}{\pi^{2} k_{0} F^{3}} \sum_{p=0}^{\infty} A_{2 p+1}^{(2 p+1)} \frac{s e_{2 p+1}\left(\frac{\pi}{2},-q\right) S e_{2 p+1}^{\prime}(0,-q)}{p_{2 p+1}^{\prime} I_{2 p+1}\left(\frac{k_{0} b}{\beta \gamma}\right)} \sum_{l=0}^{\infty} A_{2 l+1}^{(2 l+1)} \frac{s e_{2 l+1}^{\prime}(0,-q) s e_{2 l+1}\left(\frac{\pi}{2},-q\right)}{p_{2 l+1}^{\prime} I_{2 l+1}\left(\frac{k_{0} b}{\beta \gamma}\right)} L_{p, l}^{d_{y}}\left(\mu_{b} \rightarrow \infty\right), \\
Z_{\perp, x}^{d}=\frac{Z_{s} \beta \sqrt{2}}{\pi^{2} k_{0} F^{3}} \sum_{p=0}^{\infty} B_{2 p+1}^{(2 p+1)} \frac{c e_{2 p+1}^{\prime}\left(\frac{\pi}{2},-q\right) C e_{2 p+1}(0,-q)}{s_{2 p+1}^{\prime} I_{2 p+1}\left(\frac{k_{0} b}{\beta \gamma}\right)} \sum_{l=0}^{\infty} B_{2 l+1}^{(2 l+1)} \frac{c e_{2 l+1}(0,-q) c e_{2 l+1}^{\prime}\left(\frac{\pi}{2},-q\right)}{s_{2 l+1}^{\prime} I_{2 l+1}\left(\frac{k_{0} b}{\beta \gamma}\right)} L_{p, l}^{d_{x}}\left(\mu_{b} \rightarrow \infty\right), \\
Z_{\perp, y}^{q}=\frac{Z_{s} \beta \sqrt{2}}{\pi^{2} k_{0} F^{3}} \sum_{p=0}^{\infty} A_{2 p}^{(2 p)} \frac{c e_{2 p}\left(\frac{\pi}{2},-q\right) C e_{2 p}^{\prime \prime}(0,-q)}{p_{2 p}^{\prime} I_{2 p}\left(\frac{k_{0} b}{\beta \gamma}\right)} \sum_{l=0}^{\infty} A_{2 l}^{(2 l)} \frac{c e_{2 l}(0,-q) c e_{2 l}\left(\frac{\pi}{2},-q\right)}{p_{2 l}^{\prime} I_{2 l}\left(\frac{k_{0} b}{\beta \gamma}\right)} L_{p, l}^{m}\left(\mu_{b} \rightarrow \infty\right), \\
Z_{\perp, x}^{q}=\frac{Z_{s} \beta \sqrt{2}}{\pi^{2} k_{0} F^{3}} \sum_{p=0}^{\infty} A_{2 p}^{(2 p)} \frac{c e_{2 p}^{\prime \prime}\left(\frac{\pi}{2},-q\right) C e_{2 p}(0,-q)}{p_{2 p}^{\prime} I_{2 p}\left(\frac{k_{0} b}{\beta \gamma}\right)} \sum_{l=0}^{\infty} A_{2 l}^{(2 l)} \frac{c e_{2 l}(0,-q) c e_{2 l}\left(\frac{\pi}{2},-q\right)}{p_{2 l}^{\prime} I_{2 l}\left(\frac{k_{0} b}{\beta \gamma}\right)} L_{p, l}^{m}\left(\mu_{b} \rightarrow \infty\right) .
\end{gathered}
$$

Moreover, by substituting $p^{\prime}$ and $s^{\prime}$ with the corresponding Mathieu functions, we have

$$
\begin{aligned}
& Z_{\|}=\frac{Z_{s} \sqrt{2}}{\pi^{2} F} \sum_{p=0}^{\infty}(-1)^{p} A_{0}^{(2 p)} A_{2 p}^{(2 p)} \frac{C e_{2 p}(0,-q)}{c e_{2 p}(0,-q) I_{2 p}\left(\frac{k_{0} b}{\beta \gamma}\right)} \sum_{l=0}^{\infty}(-1)^{l} A_{0}^{(2 l)} A_{2 l}^{(2 l)} \frac{1}{I_{2 l}\left(\frac{k_{0} b}{\beta \gamma}\right)} L_{p, l}^{m}\left(\mu_{b} \rightarrow \infty\right), \\
& Z_{\perp, y}^{d}=\frac{Z_{s} \beta \sqrt{2}}{\pi^{2} k_{0} F^{3}} q \sum_{p=0}^{\infty}(-1)^{p} A_{1}^{(2 p+1)} A_{2 p+1}^{(2 p+1)} \frac{S e_{2 p+1}^{\prime}(0,-q)}{s e_{2 p+1}^{\prime}(0,-q) I_{2 p+1}\left(\frac{k_{0} b}{\beta \gamma}\right)} \sum_{l=0}^{\infty}(-1)^{l} A_{1}^{(2 l+1)} A_{2 t+1}^{(2 l+1)} \frac{1}{I_{2 l+1}\left(\frac{k_{0} b}{\beta \gamma}\right)} L_{p, l}^{d_{y}}\left(\mu_{b} \rightarrow \infty\right), \\
& Z_{\perp, x}^{d}=\frac{Z_{s} \beta \sqrt{2}}{\pi^{2} k_{0} F^{3}} q \sum_{p=0}^{\infty}(-1)^{p+1} B_{1}^{(2 p+1)} B_{2 p+1}^{(2 p+1)} \frac{C e_{2 p+1}(0,-q)}{c e_{2 p+1}(0,-q) I_{2 p+1}\left(\frac{k_{0} b}{\beta \gamma}\right)} \sum_{l=0}^{\infty}(-1)^{l+1} B_{1}^{(2 l+1)} B_{2 l+1}^{(2 l+1)} \frac{1}{I_{2 l+1}\left(\frac{k_{0} b}{\beta \gamma}\right)} L_{p, l}^{d_{x}}\left(\mu_{b} \rightarrow \infty\right), \\
& Z_{\perp, y}^{q}=\frac{Z_{s} \beta \sqrt{2}}{\pi^{2} k_{0} F^{3}} \sum_{p=0}^{\infty}(-1)^{p} A_{0}^{(2 p)} A_{2 p}^{(2 p)} \frac{C e_{2 p}^{\prime \prime}(0,-q)}{c e_{2 p}(0,-q) I_{2 p}\left(\frac{k_{0} b}{\beta \gamma}\right)} \sum_{l=0}^{\infty}(-1)^{l} A_{0}^{(2 l)} A_{2 l}^{(2 l)} \frac{1}{I_{2 l}\left(\frac{k_{0} b}{\beta \gamma}\right)} L_{p, l}^{m}\left(\mu_{b} \rightarrow \infty\right), \\
& Z_{\perp, x}^{q}=\frac{Z_{s} \beta \sqrt{2}}{\pi^{2} k_{0} F^{3}} \sum_{p=0}^{\infty}(-1)^{p} A_{0}^{(2 p)} A_{2 p}^{(2 p)} \frac{c e_{2 p}^{\prime \prime}\left(\frac{\pi}{2},-q\right) C e_{2 p}(0,-q)}{c e_{2 p}(0,-q) c e_{2 p}\left(\frac{\pi}{2},-q\right) I_{2 p}\left(\frac{k_{0} b}{\beta \gamma}\right)} \sum_{l=0}^{\infty}(-1)^{l} A_{0}^{(2 l)} A_{2 l}^{(2 l)} \frac{1}{I_{2 l}\left(\frac{k_{0} b}{\beta \gamma}\right)} L_{p, l}^{m}\left(\mu_{b} \rightarrow \infty\right) .
\end{aligned}
$$

Since, as we have already said, all the coefficients of the expansions are negligible except the diagonal ones, we can keep only the terms $p=l=0$ in the sums. Moreover, when $q \rightarrow 0$ we have 


$$
\begin{gathered}
A_{0}^{(0)}=c e_{0}(0,0)=c e_{0}\left(\frac{\pi}{2}, 0\right)=C e_{0}(0,0)=\frac{1}{\sqrt{2}}, \\
A_{1}^{(1)}=B_{1}^{(1)}=c e_{1}(0,0)=C e_{1}(0,0) \\
=s e_{1}^{\prime}(0,0)=S e_{1}^{\prime}(0,0)=1, \\
C e_{0}^{\prime \prime}(0,-q \rightarrow 0)=c e_{0}^{\prime \prime}\left(\frac{\pi}{2},-q \rightarrow 0\right)=\sqrt{2} q,
\end{gathered}
$$

so that the impedances become

$$
\begin{gathered}
Z_{\|}=\frac{Z_{s} \sqrt{2}}{4 \pi^{2} F I_{0}^{2}\left(\frac{k_{0} b}{\beta \gamma}\right)} L_{0,0}^{m}\left(\mu_{b} \rightarrow \infty\right), \\
Z_{\perp, y, x}^{d}=\frac{Z_{s} \beta \sqrt{2}}{\pi^{2} k_{0} F^{3} I_{1}^{2}\left(\frac{k_{0} b}{\beta \gamma}\right)} q L_{0,0}^{d_{y, x}}\left(\mu_{b} \rightarrow \infty\right), \\
Z_{\perp, y, x}^{q}=\frac{Z_{s} \beta \sqrt{2}}{2 \pi^{2} k_{0} F^{3} I_{0}^{2}\left(\frac{k_{0} b}{\beta \gamma}\right)} q L_{0,0}^{m}\left(\mu_{b} \rightarrow \infty\right) .
\end{gathered}
$$

Finally we have also that when since $\mu_{b} \rightarrow \infty$ the hypergeometric functions tend to 1 so that

$$
\begin{aligned}
& L_{0,0}^{m}=2 \sqrt{2} \pi e^{-\mu_{b}}=2 \sqrt{2} \pi e^{-\operatorname{arccosh}\left(\frac{a}{F}\right)}, \\
& L_{0,0}^{d_{y, x}}=\sqrt{2} \pi e^{-\mu_{b}}\left(1 \pm \frac{e^{-2 \mu_{b}}}{2}\right)=\sqrt{2} \pi e^{-\operatorname{arccosh}\left(\frac{a}{F}\right)} .
\end{aligned}
$$

When $F \rightarrow 0$ and $a=b$

$$
\lim _{F \rightarrow 0} e^{-\operatorname{arccosh}\left(\frac{b}{F}\right)}=\frac{F}{2 b}
$$

and, by using Eq. (5), we finally obtain

$$
\begin{aligned}
Z_{\|} & =\frac{Z_{s}}{2 \pi b I_{0}^{2}\left(\frac{k_{0} b}{\beta \gamma}\right)}, \\
Z_{\perp, y, x}^{d} & =\frac{Z_{s} k_{0}}{4 \pi b \beta \gamma^{2} I_{1}^{2}\left(\frac{k_{0} b}{\beta \gamma}\right)}, \\
Z_{\perp, y, x}^{q} & =\frac{Z_{s} k_{0}}{4 \pi^{2} b \beta \gamma^{3} I_{0}^{2}\left(\frac{k_{0} b}{\beta \gamma}\right)},
\end{aligned}
$$

which coincide with the impedances in a circular pipe.

[1] L. Palumbo, V. G. Vaccaro, and M. Zobov, Wake fields and impedance, CERN Technical Report No. CERN 95-06, 1995 [arXiv:physics/0309023].
[2] V. G. Vaccaro, Longitudinal instabilities of a coasting beam above transition, due to the action of lumped discontinuities, CERN Technical Report No. ISR-RF/66-35, 1966.

[3] A. M. Sessler and V. G. Vaccaro, Longitudinal instabilities of azimuthally uniform beam in circular vacuum chamber with walls of arbitrary electrical properties, CERN Technical Report No. CERN 67-2, 1967.

[4] A. W. Chao, Physics of Collective Beam Instabilities in High Energy Accelerators (John Wiley \& Sons, New York, 1993).

[5] K. Y. Ng, Physics of Intensity Dependent Beam Instabilities (World Scientific, Singapore, 2006).

[6] K. L. F. Bane and G. Stupakov, Resistive wall wakefield in the LCLS undulator, in Proceedings of the 21st Particle Accelerator Conference, Knoxville, TN, 2005 (IEEE, Piscataway, NJ, 2005), p. 3390.

[7] M. Migliorati, E. Belli, and M. Zobov, Impact of the resistive wall impedance on beam dynamics in the Future Circular $e^{+} e^{-}$Collider, Phys. Rev. Accel. Beams 21, 041001 (2018).

[8] E. Belli, P.C. Pinto, G. Rumolo, A. Sapountzis, T. Sinkovits, M. Taborelli, B. Spataro, M. Zobov, G. Castorina, and M. Migliorati, Electron cloud buildup and impedance effects on beam dynamics in the Future Circular $e^{+} e^{-}$Collider and experimental characterization of thin TiZrV vacuum chamber coatings, Phys. Rev. Accel. Beams 21, 111002 (2018).

[9] E. Belli, G. Castorina, M. Migliorati, G. Rumolo, B. Spataro, and M. Zobov, Single bunch instabilities in FCC-ee, in 9th International Particle Accelerator Conference (IPAC18), p. 3336, Vancouver, BC, Canada, 2018, http://accelconf .web.cern.ch/AccelConf/ipac2018/.

[10] E. Belli, G. Castorina, M. Migliorati, A. Novokhatski, S. Persichelli, B. Spataro, and M. Zobov, Coupling impedances and collective effects for FCC-ee, in 8th International Particle Accelerator Conference (IPAC17), p. 3734, Copenhagen, Denmark, 2017, http://accelconf .web.cern.ch/AccelConf/ipac2017/.

[11] M. Migliorati, S. Persichelli, H. Damerau, S. Gilardoni, S. Hancock, and L. Palumbo, Beam-wall interaction in the CERN Proton Synchrotron for the LHC upgrade, Phys. Rev. Accel. Beams 16, 031001 (2013).

[12] S. Persichelli, M. Migliorati, N. Biancacci, S. Gilardoni, E. Métral, and B. Salvant, Transverse beam coupling impedance of the CERN Proton Synchrotron, Phys. Rev. Accel. Beams 19, 041001 (2016).

[13] M. Migliorati, S Aumon, E. Koukovini-Platia, A. Huschauer, E Métral, G. Sterbini, and N. Wang, Instability studies at the CERN Proton Synchrotron during transition crossing, Phys. Rev. Accel. Beams 21, 120101 (2018).

[14] A. Lutman, R. Vescovo, and P. Craievich, Electromagnetic field and short-range wake function in a beam pipe of elliptical cross section, Phys. Rev. Accel. Beams 11, 074401 (2008).

[15] K.Yokoya, Resistive wall impedance of beam pipes of general cross section, Part. Accel. 41, 221 (1993); KEKPreprint-92-196.

[16] F. Ruggiero, Resistive wall impedance as derivative of the electric capacitance for a beam pipe of arbitrary cross section, CERN Technical Report No. CERN-SL-95-95(AP), 1995. 
[17] R. L. Gluckstern, J. van Zeijts, and B. Zotter, Coupling impedance of beam pipes of general cross section, Phys. Rev. E 47, 656 (1993).

[18] A. Piwinski, Impedances in lossy elliptical vacuum chambers, DESY Technical Report No. DESY-94-068, 1994.

[19] L. Palumbo and V. G. Vaccaro, The coupling impedance between a circular beam and a lossy elliptic vacuum chamber in particle accelerators, Nuovo Cimento Soc. Ital. Fis. 89A, 243 (1985).

[20] U. Niedermayer, O. Boine-Frankenheim, and H. De Gersem, Space charge and resistive wall impedance computation in the frequency domain using the finite element method, Phys. Rev. Accel. Beams 18, 032001 (2015).

[21] S. Persichelli, N. Biancacci, M. Migliorati, L. Palumbo, and V. G. Vaccaro, Electromagnetic fields and Green's functions in elliptical vacuum chambers, Phys. Rev. Accel. Beams 20, 101004 (2017).

[22] M. Migliorati, N. Biancacci, M. R. Masullo, L. Palumbo, and V. G. Vaccaro, Space charge impedance and electromagnetic fields in elliptical vacuum chambers, Phys. Rev. Accel. Beams 21, 124201 (2018).

[23] M. Migliorati, N. Biancacci, L. Palumbo, and V. G. Vaccaro, Longitudinal and quadrupolar coupling impedance of an elliptical vacuum chamber with finite conductivity in terms of Mathieu functions, in 9th International Particle Accelerator Conference (IPAC18), p. 3040, Vancouver, BC, Canada, 2018, http://accelconf.web.cern .ch/AccelConf/ipac2018/.

[24] N.W. McLachlan, Theory and Application of Mathieu Functions (Dover Publications, New York, 1964).

[25] Here we use the transverse impedance definition of Ref. [26], compatible, for example with the code IW2D [27]. In other papers, as in [5], there is an additional $1 / \beta$ multiplying factor that has to be taken into account for beam dynamics studies.

[26] R L Gluckstern, Analytic Methods for Calculating Coupling Impedances, CERN Yellow Reports: Monographs (CERN, Geneva, 2000).

[27] N. Mounet, The LHC transverse coupled bunch instability, Ph.D. thesis, École Polytechnique Fédérale de Lausanne, Lausanne, Switzerland, January 2012.

[28] K. Bane and G. Stupakov, Using surface impedance for calculating wakefields in flat geometry, Phys. Rev. Accel. Beams 18, 034401 (2015).
[29] B. Henrist, N. Hilleret, C. Scheuerlein, and M. Taborelli, The secondary electron yield of TiZr and TiZrV non-evaporable getter thin film coatings, Appl. Surf. Sci. 172, 95 (2001).

[30] C. Zannini, Electromagnetic simulations of CERN accelerator components. Ph.D. thesis, École Polytechnique Fédérale de Lausanne, Lausanne, Switzerland, June 2013.

[31] L. Vos, The impedance of multilayer vacuum chambers, CERN Technical Report No. CERN-AB-2003-093-ABP, 2003.

[32] L. Vos, The transverse impedance of a cylindrical pipe with arbitrary surface impedance, CERN Technical Report No. CERN-AB-2003-005-ABP, 2003.

[33] A. Koschik, F. Caspers, E. Métral, L. Vos, and B. W. Zotter, Transverse resistive wall impedance and wake function with inductive bypass, in Proceedings of the 9th European Particle Accelerator Conference, Lucerne, 2004 (EPS-AG, Lucerne, 2004), pp. 1876-1878, WEPLT023 [http:// accelconf.web.cern.ch/AccelConf/e04/].

[34] A. Koschik, Transverse resistive wall wake function with inductive bypass, CERN Technical Report No. CERN-AB2003-088-ABP, 2003.

[35] A. Danisi, C. Zannini, R. Losito, A. Masi, and B. Salvant, Theoretical analysis of metamaterial insertions for resistive-wall beam-coupling impedance reduction, In Proc. 5th Int. Particle Accelerator Conf. (IPAC'14), pp. 1695-1697, Dresden, Germany, June 2014. tupri055.

[36] C. Zannini and T Rijoff, TL wall: transmission line model for wall impedance calculations, CERN, Geneva, Switzerland, 18 February 2019. Presented at the HSC section meeting. https://indico.cern.ch/event/795854/ contributions/3306468/.

[37] https://www.cst.com.

[38] C. Zannini, N. Biancacci, T. L. Rijoff, and G. Rumolo, Electromagnetic simulations for non-ultrarelativistic beams and application to the CERN low energy machines, In Proc. 5th International Particle Accelerator Conference (IPAC'14), Dresden, Germany, June 15-20, 2014, number 5 in International Particle Accelerator Conference, pp. 1718-1720, Geneva, Switzerland, July 2014. JACoW. https://doi.org/10.18429/JACoW-IPAC2014-TUPRI063.

[39] I. S. Gradshteyn and I. M. Ryzhik, Table of Integrals, Series, and Products (Elsevier/Academic Press, Amsterdam, 2007). 